\title{
Análise de perfil hematológico e exame coproparasitológico de cães em relação ao indicativo da presença de verminoses em um canil em Porto Velho - RO
}

\author{
Analysis of the hematological profile and the coproparasitological examination of dogs in relation \\ to the indication of the presence of verminosis in a kennel in Porto Velho - RO \\ Análisis del perfil hematológico y el examen coproparasitológico de perros en relación a la \\ indicación de presencia de verminosis en una perrera en Porto Velho - RO
}

Recebido: 08/07/2021 | Revisado: 15/07/2021 | Aceito: 30/07/2021 | Publicado: 18/08/2021

\author{
Thiago Vaz Lopes \\ ORCID: https://orcid.org/0000-0002-3192-1908 \\ Universidade Federal do Acre, Brasil \\ E-mail: Thiagovlopes@hotmail.com \\ Clívia de Melo Pessôa \\ ORCID: https://orcid.org/0000-0002-5405-873X \\ Centro Universitário Aparício Carvalho, Brasil \\ E-mail: cliviademelo63@gmail.com \\ Paloma Gabrielle Lopes Leão \\ ORCID: https://orcid.org/0000-0002-9657-1910 \\ Centro Universitário Aparício Carvalho, Brasil \\ E-mail: palomaleaomedvet@gmail.com \\ João Gustavo da Silva Garcia de Souza \\ ORCID: https://orcid.org/0000-0002-5899-2490 \\ Centro Universitário Aparício Carvalho, Brasil \\ E-mail: joaogarciadesouza27@gmail.com \\ Sandro de Vargas Schons \\ ORCID: https://orcid.org/0000-0001-9811-5356 \\ Universidade Federal de Rondônia, Brasil \\ E-mail: sandroschons@unir.br \\ Fernando Andrade Souza \\ ORCID: https://orcid.org/0000-0002-9474-9404 \\ Universidade Federal do Paraná, Brasil \\ E-mail: femedvet@yahoo.com.br \\ Núbia Venâncio da Costa \\ ORCID: https://orcid.org/0000-0002-9104-8813 \\ Centro Universitário Aparício Carvalho \\ E-mail: ubiavenancio26@gmail.com \\ Thaís de Almeida Souza \\ ORCID: https://orcid.org/0000-0002-2722-9601 \\ Centro Universitário Aparício Carvalho \\ E-mail: thaís_medvet@hotmail.com
}

\begin{abstract}
Resumo
Em muitos casos os tutores desconhecem as questões de cuidados com a saúde dos animais e não promovem nenhum tipo de protocolo antiparasitário ou ainda efetuam de forma inadequada, o que tende a ser um fator zoonótico, tornando então de extrema importância o tratamento adequado. Dessa forma, o exame coproparasitológico de fezes (EPF) juntamente com a avaliação do hemograma, possibilitam diagnosticar um grande número de enfermidades parasitárias. O presente estudo tem como objetivo avaliar os resultados do perfil hematológico e coproparasitológico no indicativo para verminose em animais provenientes de um canil em Porto Velho, Rondônia. O trabalho foi realizado em um canil de Porto-Velho, Rondônia, com a coleta de sangue e fezes, de cães de diferentes raças, idade e sexo. Pelo exame hematológico, foi possível constatar que 12,5\% (2/16) dos animais analisados apresentaram eosinofilia, logo, 87,5\% (14/16) do grupo não ultrapassaram o limite dos valores de referência de caninos para eosinófilos (150-1.250 eosinófilos/ $\mu$ l), utilizados em um hospital veterinário de uma instituição de ensino superior. Os resultados do exame coproparasitológico indicaram que 18,75\% (3/16) das amostras de fezes, encontravam-se com ovos de Ancylostoma sp., $\log$, $81,25 \%$ (13/16) não apresentaram verminoses. Conclui-se que houve divergência entre os métodos, no qual o exame coproparasitológico por meio da técnica de Willis-Mollay foi evidenciado como mais fidedigno na avaliação de
\end{abstract}


parasitose, em contrapartida, o aparelho para exame hematológico automatizado não foi efetivo, indicando os diferentes resultados.

Palavras-chave: Eosinofilia; Hemograma; Parasitos; Zoonose.

\begin{abstract}
In many cases, guardians are unaware of the issues of animal health care and do not promote any type of antiparasitic protocol or even perform it inappropriately, which tends to be a zoonotic factor, thus making adequate treatment extremely important. Thus, the fecal stool examination (EPF) together with the evaluation of the blood count, make it possible to diagnose a large number of parasitic diseases. The present study aims to evaluate the results of the hematological and coproparasitological profile in the indicative for verminosis in animals from a kennel in Porto Velho, Rondônia. The work was carried out in a kennel in Porto-Velho, Rondônia, with the collection of blood and feces from dogs of different breeds, age and sex. The hematological examination showed that $12.5 \%(2 / 16)$ of the animals analyzed presented eosinophilia, therefore, $87.5 \%$ (14/16) of the group did not exceed the limit of reference values for canines for eosinophils $(150-1,250$ eosinophils/ $\mu$ l) used in a veterinary hospital of a higher education institution. The results of the coproparasitological examination indicated that $18.75 \%(3 / 16)$ of the fecal samples had Ancylostoma sp. eggs, therefore, $81.25 \%(13 / 16)$ did not present verminosis. It is concluded that there was a divergence between the methods, in which the coproparasitological examination using the Willis-Mollay technique was evidenced as more reliable in the evaluation of parasitosis, on the other hand, the device for automated hematological examination was not effective, indicating the different results.
\end{abstract}

Keywords: Eosinophilia; Blood count; Parasites; Zoonosis.

\title{
Resumen
}

En muchos casos, los tutores desconocen la problemática del cuidado de la salud animal y no promueven ningún tipo de protocolo antiparasitario o incluso lo realizan de manera inapropiada, lo que tiende a ser un factor zoonótico, por lo que el tratamiento adecuado es de suma importancia. Así, el examen de heces fecales (EPF) junto con la evaluación del hemograma, permiten diagnosticar un gran número de enfermedades parasitarias. El presente estudio tiene como objetivo evaluar los resultados del perfil hematológico y coproparasitológico en el indicativo de verminosis en animales de una perrera en Porto Velho, Rondônia. El trabajo se llevó a cabo en una perrera en Porto-Velho, Rondônia, con la recogida de sangre y heces de perros de diferentes razas, edad y sexo. El examen hematológico mostró que el 12,5\% (2/16) de los animales analizados presentaban eosinofilia, por lo que el 87,5\% (14/16) del grupo no superó el límite de valores de referencia para caninos para eosinófilos (150 - 1.250 eosinófilos / $\mu \mathrm{l})$ utilizados en un hospital veterinario de una institución de educación superior. Los resultados del examen coproparasitológico indicaron que el 18,75\% (3/16) de las muestras fecales tenían huevos de Ancylostoma sp., por lo que el 81,25\% (13/16) no presentaba verminosis. Se concluye que hubo divergencia entre los métodos, en los cuales el examen coproparasitológico mediante la técnica de Willis-Mollay se evidenció como más confiable en la evaluación de parasitosis, por otro lado, el dispositivo para examen hematológico automatizado no resultó efectivo, indicando los diferentes resultados.

Palabras clave: Eosinofilia; Hemograma; Parásitos; Zoonosis.

\section{Introdução}

O crescente contato do cão com o homem impõe a necessidade a alargar os cuidados com a saúde desses animais, na qual mesmo com todos os benefícios ainda representam uma importante fonte de agentes zoonóticos, incluindo parasitos, bactérias e fungos. (Geffray, 1999; Plant et al. 1996). Em muitos casos os tutores desconhecem essas questões, e com isso não promovem nenhum tipo de protocolo antiparasitário em seus animais ou quando utilizam deste controle, efetuam de forma inadequada e como consequência verifica-se uma resistência anti-helmíntica, e não eliminação das verminoses (Bremm, 2007).

O exame coproparasitológico de fezes (EPF) é a base para o diagnóstico de um grande número de enfermidades causadas por endoparasitos, e se baseia na detecção do agente em suas diferentes fases, como em ovos e larvas de helmintos ou de cistos ou oocistos no caso de protozoários, em amostras de fezes, sendo muito utilizado em estudos para a análise de contaminação populacional de demarcados lugares, como em praças públicas (Antunes et al. 2020; Barros, 2018; Evaristo et al. 2018) e Hospitais veterinários (Ferreira et al. 2020; Torres et al. 2020). Os métodos coproparasitológicos podem ser realizados com amostras fecais frescas ou preservado. Para a fixação das amostras é conhecido uma abrangente classe de fixadores, voltados à preservação das características morfológicas dos parasitos, aumentando a chance de que eles sejam detectados e corretamente identificados (Garcia, 1995). 
O hemograma é um instrumento importante na clínica de cães e gatos, podendo ser utilizado como exame de triagem, em diagnóstico, prognóstico e monitoramento. Na maioria dos casos em forma isolada o hemograma apresenta-se como um recurso limitado na busca pelo diagnóstico (Gonzales, 2005).

O hemograma inclui: contagem de glóbulos vermelhos com índices eritrocitários; contagem de glóbulos brancos; contagem de plaquetas e avaliação da morfologia celular, dentre os glóbulos brancos temos o eosinófilo, também participa na regulação da resposta alérgica e inflamatória. Outra função importante dos eosinófilos é o controle de infecções parasitárias, por um processo de degranulação e com isso uma ação parasiticida (Aguiar, 2010; Lopes et.al., 2007; Lopes, Biondo, \& Santos, 2007; Nelson \& Couto, 2006; Rebar, 2003).

A avaliação dos exames e diagnostico de verminose, permite salientar as pessoas que possuem convívio próximo aos animais, à probabilidade de se contaminarem a fim de deixar a população consciente, sobre a educação sanitária concomitante aos bons cuidados com o objetivo de reduzir a incidência destas zoonoses ao mínimo (Cardozo, 2013).

Sendo assim, o intuito desse trabalho consiste em avaliar os resultados da porcentagem de eosinófilos frente a carga parasitária encontrada no coproparasitológico, em animais provenientes de um canil no município de Porto Velho, Rondônia.

\section{Material e Métodos}

Foram coletadas um total de 16 ( $\mathrm{n}=16$ ) amostras de sangue e fezes, no mês outubro de 2019, em um canil localizado em Porto-Velho, Rondônia. As amostras foram coletas de cães, de diferentes raças, idade e sexo, totalizando 16 amostras. As coletas foram no período da manhã, sendo armazenadas em coletor universal e as de sangue em tubo de ensaio. $\mathrm{O}$ trabalho foi submetido ao Conselho de ética no uso de animais, da Universidade Federal do Acre e aprovado com número de protocolo 13/2017 CEUA-UFAC.

O exame de coproparasitológico das fezes procedeu-se pela técnica de Willis-Mollay (1921), baseada na flutuação de ovos leves em solução saturada de $\mathrm{NaCl}$ Logo. Foram obtidas 16 lâminas em todo o experimento, analisadas por microscopia óptica, em objetiva de 10x. O resultado se deu pela identificação morfológica de ovos e oocistos nas bordas das lamínulas.

Para o exame de sangue utilizou-se seringas de $3 \mathrm{ml}$ com agulhas descartáveis e tubos de ensaio para coleta, além de gazes e álcool para a higienização da pele do animal. Sendo encaminhadas para o processamento por hematologia automatizado, bem como realizou-se a leitura das lâminas para o leucograma diferencial.

As lâminas foram feitas por meio da técnica de esfregaço e fixação, onde se coloca uma gota do material, no caso sangue, na região próxima a parte fosca da lâmina. Depois disso, é utilizado outra lâmina para a realização do esfregaço, onde se posiciona a lâmina em $45^{\circ}$ sob a superfície da lâmina com o sangue, arrastando o material em direção a outra extremidade (Amedee \& Dhurandhar, 2001; Kline, 1988).

Feito esse processo, foi então realizado a fixação do sangue na lâmina com a utilização do Kit Panótico rápido LB Laborclin para Laboratórios Ltda. Este tipo de fixação é baseado no princípio de coloração hematológica idealizado por Romanowsky, onde se utilizada um fixador e dois corantes, por meio de imersão de 5 segundo cada. Após a última imersão, já é possível a realização da leitura, para a procura do que a pesquisa se mostra focada, como os leucócitos ou algum parasito intracelular, por exemplo (Brandão \& Hagiwara, 2002).

\section{Resultados e Discussão}

Com os resultados obtidos da interpretação dos dois exames, foi possível observar que ocorreram alterações hematológicas nos animais com a presença de verminoses. Dessa forma, verificou-se as modificações causadas por verminoses, no perfil hematológico dos animais estudados, assim como o observado por Ferreira et al. (2014). 
Pelo exame hematológico, foi possível constatar que 12,5\% (2/16) dos animais analisados apresentaram eosinofilia, logo, $87,5 \%$ (14/16) do grupo não ultrapassaram o limite dos valores de referência de caninos para eosinófilos (100-1.250 eosinófilos/ $\mu 1$ ), o que diverge com o achado de Silva et al. (2010), que observaram um leve aumento nos 49 cães parasitados por helmintos em seu estudo. Os hemogramas obtidos, também expressaram que 6,25\% (1/16) apresentaram $\geq 800$ eosinófilos/ $\mu$ l, além disso, 12,5\% (2/16) apresentaram $\geq 700$ eosinófilos/ $\mu 1$, assim como, 12,5\% (2/16) apresentaram $\geq 600$ eosinófilos/ $\mu 1$, com os outros 56,25\% (9/16) apresentando $\geq 500$ eosinófilos/ $\mu 1$ assim como observado na Tabela 1.

Tabela 1 - Tabela para demonstrar os valores de eosinófilos encontrados, suas porcentagens por animais e o seu valor de referência para caninos.

\begin{tabular}{|c|c|c|c|}
\hline Quantidade de animais & Porcentagem (\%) & $\begin{array}{c}\text { Valores de Eosinófilos }(\mu \mathrm{l}) \\
\text { achados }\end{array}$ & Valores de Referência $(\mu \mathrm{l})$ \\
\hline 2 & 12,5 & $\leq 1.350$ & $100-1.250$ \\
\hline 1 & 6,25 & $\geq 800$ & $100-1.250$ \\
\hline 2 & 12,5 & $\geq 700$ & $100-1.250$ \\
\hline 2 & 12,5 & $\geq 600$ & $100-1.250$ \\
\hline 9 & 56,25 & $\geq 500$ & $100-1.250$ \\
\hline 16 & 99,98 & - & - \\
\hline
\end{tabular}

Fonte: Autores.

Os resultados do exame coproparasitológico indicaram que 18,75\% (3/16) das amostras de fezes, encontravam-se contaminadas por ovos de Ancylostoma sp., logo, 81,25\% (13/16) não apresentaram verminoses, como o demonstrado na Tabela 2. Comparando os resultados dos dois exames utilizados, pôde-se observar alterações hematológicas em apenas alguns dos animais com a presença de parasitos do grupo estudado.

Tabela 2 - Número de animais estudados que continham a presença de ovos de endoparasitos em suas fezes.

\begin{tabular}{cccc}
\hline Contaminado & $\mathbf{N}^{\circ}$ de animais & Porcentagem (\%) & Parasito \\
\hline Sim & 3 & 18,75 & Ancylostoma sp. \\
Não & 13 & 81,25 & - \\
\hline- & 16 & 100 & - \\
\hline
\end{tabular}

Fonte: Autores.

O Atual trabalho se mostrou divergente com o que foi encontrado por Ferreira et al. (2014), em que a análise hematológica se demonstrou um importante indicador de parasitismo quando comparado ao coproparasitológico, no qual o mesmo evidenciou tanto quanto o resultado obtido no hemograma, devido alteração significativa na contagem de eosinófilos em comparação a quantidade de parasitos, encontrados no exame coproparasitológico de equinos e asininos da região de São LuísMA.

O limite dos valores de referência para caninos em relação aos eosinófilos utilizados são os de Meinkoth e Clinkenbeard (2000), onde o fisiológico está entre 100-1.250 eosinófilos/ $\mu$ l. Logo, em concordância com o atual trabalho, a pesquisa de Silva 
et al. (2010) sobre as alterações hematológicas ocasionadas por helmintos avaliada em 42 cães, apontou que $48 \%$ dos animais infectados por Ancylostoma sp. e Toxocara sp. apresentavam eosinofilia, sendo a média contabilizada em 1.302 eosinófilos, considerando então, que essa alteração pode ser indicativa da presença de parasitoses.

Quando analisado e comparado a eosinofilia de animais afetados com helmintos com os achados em animais acometidos com protozoários, também não se observa grande diferença entre ambos. Fernandes et al. (2013) relatou que nem todos os animais acometidos por Babesia spp. demonstram anormalidades no leucograma, corroborando com Taboada \& Merchant (1997), que dizem que as anormalidades leucocitárias são achados muito imprecisos, visto que variam de animal para animal.

Dessa forma, em comparação a outros estudos, como o feito por Ferreira et al. (2014) é possível avaliar a nítida discordância entre os parâmetros que consideram um animal acometido por parasitose, frente à carga eosinofílica. Lunardon et al. (2016) relata que a avaliação somente por meio de achados eosinofílicos em hemogramas não é um marcador suficiente para conseguir diagnosticar um animal com endoparasitos, sendo necessário a utilização do coproparasitológico em conjunto com o mesmo, afim de realizar um diagnóstico preciso.

\section{Conclusão}

Conclui-se que houve divergência entre os métodos aplicados no presente artigo, no qual o exame coproparasitológico por meio da técnica de Willis-Mollay (1921) foi evidenciado como mais fidedigno na avaliação de parasitose, quando comparado com a porcentagem de eosinófilos encontrados no leucograma.

Mesmo sendo um número muito baixo de animais analisados, consegue se ter uma breve noção de que a análise por meio da quantidade de eosinófilos para a averiguação de parasitos não é totalmente eficaz. Com isso, a técnica de flutuação continua sendo uma forma muito mais precisa para a constatação de uma possível infestação parasitária.

\section{Referências}

Aguiar, F. G. P. L. (2010). O Hemograma no Cão e Contribuição para a Sua Caracterização no Cão da Serra da Estrela, Variedade de Pêlo Comprido. Universidade Lusófona de Humanidades e Tecnologias, Lisboa.

Amedee, R. G. \& Dhurandhar, N. R. (2001). Fine-needle aspiration biopsy. Laryngoscope, 111(9): 1551-1557.

Antunes, T. A. (2020). Frequência de helmintos em amostras fecais de cães em praças públicas de Pelotas-RS. PUBVET. 14(8): 1-6.

Barros, B. A. F. (2018). Ocorrência de parasitas gastrintestinais em fezes de cães coletadas em vias públicas do município de Valença - RJ. PUBVET. 12(9): 19.

Brandão, L. P. \& Hagiwara, M. K. (2002). Babesiose canina - Revisão. Clínica Veterinária, 41: 50-59.

Cardozo, R. M. et al. (2013). Avaliação hematológica em cães errantes da região urbana de Maringá-PR. PUBVET, 7(26).

Evaristo, T. A. (2018). Prevalência de parasitos gastrointestinais em amostras fecais em praças públicas nos municípios de Pedro Osório e Cerrito, RS. Atas de Saúde Ambiental. 6: 70-84.

Fernandes, L. L., Nagayoshi, B. A. \& Barbosa, T. S. (2013). Hematologia dos cães com babesiose atendidos no hospital veterinário da Universidade de Marília - Unimar. unimar ciências, 22(1-2).

Ferreia et al. (2020). Prevalência de Helmintos Gastrointestinais em Cães atendidos no Hospital Veterinário Universitário Francisco Edilberto Uchoa Lopes da Universidade Estadual do Maranhão com Enfoque em Saúde Pública. Braz. J. of Develop., 6(6): 36192-36200.

Ferreira, G. M. S. et al. (2014). Parasitismo gastrintestinal e hematologia em equinos e asininos da mesorregião da aglomeração urbana, São Luís, Maranhão. Archives of Veterinary Science, 19(2): 22-30.

Garcia, L. S. (1995). Pros and Cons of using preservatives for O \& P fecal specimens. Clin. Microbiol. News, 17(21): 164-167.

Geffray, L. (1999). Infections associated with pets. Rev. Med. Interne, 20: 888-901.

Gonzáles, F. H. D. \& Santos, A. P. (2005). Anais do 2 Simpósio de Patologia Clínica Veterinária da Região sul do Brasil, Realizado em Porto Alegre, no ano de 2005. Porto Alegre: UFRGS. 
Research, Society and Development, v. 10, n. 10, e78101018016, 2021

(CC BY 4.0) | ISSN 2525-3409 | DOI: http://dx.doi.org/10.33448/rsd-v10i10.18016

Kline, T. S. (1988). Handbook of fine needle aspiration biopsy cytology. 2nd. New York: Churchill Livingstone.

Lopes, S., Biondo, A. \& Santos, A. (2007). Manual de Patologia Clínica Veterinária. Santa Maria: Universidade Federal de Santa Maria - Centro de Ciências Rurais.

Lunardon, T. et al. (2016). Correlação entre Eosinofilia e Parasitas Gastrintestinais em Cães. Revista Eletrônica Biociências Biotecnologia e Saúde, 15.

Mariana, B. (2007). Infecções Parasitárias por Nematódeos em Cães do Canil Municipal Santa Cruz do Sul/RS. Universidade Federal do Rio Grande do Sul. Faculdade de Veterinária e Especialização em Análises Clínicas Veterinárias, Porto Alegre.

Martins, C. R. et al. (2012). Perfil hematológico de cães (Canis familiaris) obesos e senis. Vet. Not., Uberlândia, 18(2): 62-66.

Plant, M., Zimmerman, E. M. \& Goldstein. R. A. (1996). Health hazards tohumans associated with domestic pets. Annu. Rev. Public Health, $17: 221-245$.

Rebar, A. (2003). Interpretacion del Hemograma Canino y Felino. Wilmington, 2003. Delaware: Nestlé Purina PetCare Company.

Sarles, M. P. (1929). Studies of the blood changes occurring in young and old dogs duringcutaneous and oral infection with the dog hookworm. "Aneylostoma caninum". Amer. Jour. Hyg.

Satie, K. (2006). Avaliação de Duas Técnicas Coproparasitológicas Convencionais e de um Kit Comercial na Investigação da Epidemiologia de Parasitas Gastrointestinais de Cães no Estado de São Paulo. Faculdade de Medicina Veterinária e Zootecnia Universidade Estadual Paulista- Júlio de Mesquita Filho Campus, Botucatu, São Paulo.

Silva, B. J. A. et al. (2010). Avaliação das alterações hematológicas nas infecções por helmintos e protozoários em cães (Canis lupus familiaris, Linnaeus, 1758). Neotropical Helminthology, 4(1): 37-48.

Taboada, J. \& Merchant, S. R. (1997). Infecções por protozoários e por outras causas. In: ETTINGER, S.J. \& FELDMAN, E.C. Tratado de Medicina Interna Veterinária. São Paulo: Manole, 563-565 p.

Torres, B. Á. et al. (2020). Ocorrência de parasitas gastrointestinais em cães e gatos atendidos no hospital veterinário DA Universidade Federal do Paraná setor Palotina. Archives of veterinary Science. 25(5): 25. 